\title{
LEGISLAÇÃO APLICADA À SAÚDE INDÍGENA
}

\author{
LEGILATION APPLIED TO INDIGENOUS HEALTH
}

Juliano Sguizardi ${ }^{1}$

RESUMO: Este ensaio traz à tona a razão legislativa no tocante a saúde indígena quanto à sua relação e aplicabilidade em face dos procedimentos hábeis a satisfazerem as garantias inscritas na Constituição da República Federativa do Brasil de 1988. A saúde como um todo é o pilar da vida, logo, da sociedade, base comum, tanto na seara da dignidade, quanto dos demais preceitos autodeterminantes dos indivíduos. O tema é preponderante, pois conforme consta na base de dados da FUNAI, no Brasil, possuem 305 etnias indígenas, as quais falam 274 línguas, com aproximadamente 1 milhão de indivíduos, dados verificáveis através do IBGE, sendo que estão presentes em todos os Estados da Federação do Brasil, e cada uma das etnias, com seus respectivos povos, possuem sua própria cultura, sendo que tal diversidade constitui desafio para as políticas públicas. Neste sentido, dentro da saúde pública brasileira, tem-se a Secretaria Especial de Saúde Indígena (SESAI) que é responsável pela coordenação e execução da Política Nacional de Atenção à Saúde dos Povos Indígenas, além do processo de gestão do subsistema de atenção à saúde indígena (SasiSUS), tudo vinculado ao Sistema Único de Saúde (SUS), pelo Ministério da Saúde, a qual é responsável em implementar um modelo de saúde contemporâneo em atenção às especificidades de cada etnia e também dos fatores de saúde indígena de cada região em face do fator epidemiológico e os decorrentes que afetam diretamente a saúde indígena, sensível em relação à saúde comum dos demais povos, visto que a gestão busca garantir acesso integral à saúde e, que, esse estudo demonstra a aplicabilidade jurídica da instituição Saúde Indígena em razão dos atos administrativos. Destaca-se que o trabalho fora desenvolvido através de metodologia de pesquisa mista, pelo método dedutivo e por procedimento bibliográfico, afim de quali-quantificar a relação legal para o leitor, facilitando o alcance da sistemática do funcionamento da saúde pública brasileira aplicada aos povos indígenas.

PALAVRAS CHAVE: Saúde Indígena. Políticas públicas. Legislação.

\footnotetext{
${ }^{1}$ Bacharel em Direito pela Faculdade de Colíder (2012.1), Advogado inscrito perante a OAB/MT sob o no 16.483/O, Pós-Graduação a Nível de Especialização em Saúde Indígena. Pós graduação em Gestão Pública com ênfase em Gestão Ambiental. Docente no curso de Direito Centro Universitário UNICATHEDRAL de Barra do Garças-MT.
} 
ABSTRACT: This essay brings up the legislative reason regarding indigenous health in relation to its relationship and applicability in view of the skillful procedures to satisfy the guarantees registered in the Constitution of the Federative Republic of Brazil of 1988. Health as a whole is the pillar of life, therefore, of society, a common base, both in the field of dignity, and of the other self-determining precepts of individuals. The theme is preponderant, as according to the FUNAl database, in Brazil, they have 305 indigenous ethnic groups, who speak 274 languages, with approximately 1 million individuals, data verifiable through IBGE, being present in all States of the Federation of Brazil, and each of the ethnic groups, with their respective people, has their own culture, and such diversity is a challenge for public policies. In this sense, within Brazilian public health, there is the Special Secretariat for Indigenous Health (SESAI), which is responsible for the coordination and execution of the National Policy for the Attention to Health of Indigenous Peoples, in addition to the management process of the health care subsystem. (SasiSUS), all linked to the Unified Health System (SUS), by the Ministry of Health, which is responsible for implementing a contemporary health model in attention to the specificities of each ethnic group and also of the indigenous health factors of each region in in view of the epidemiological factor and those arising that directly affect indigenous health, sensitive in relation to the common health of other peoples, since management seeks to guarantee full access to health and that this study demonstrates the legal applicability of the institution Indigenous Health due to administrative acts. It is noteworthy that the work was developed through mixed research methodology, by the deductive method and by bibliographic procedure, in order to quali-quantify the legal relationship for the reader, facilitating the reach of the Brazilian public health functioning system applied to indigenous peoples.

KEYWORDS: Indigenous Health. Public politic. Legislation. 


\section{INTRODUÇÃO}

A saúde indígena é um assunto sob demanda em sensibilização internacional, no qual a Constituição da República Federativa do Brasil de 05 de outubro de 1988 $\mathrm{CF} / 88$, em seu artigo $6^{\circ}$ instituiu de forma genérica como sendo um Direito Social de todos que aqui estão, no País, sem excluir as responsabilidades decorrentes de tratados e convenções.

Para a compreensão do texto é necessário entender que compete ao Município em cooperação técnica e financeira da União, dos Estados e do Distrito Federal a prestação de serviços de atendimento à saúde da população, logo, inclui os indígenas - art. 30, inciso VII, da CF/88.

Ocorre que a saúde do índio por ser algo sensível à problemática comum dos povos, possui atenção especial, que dentro do Ministério da Saúde há departamento específico, SESAI - Secretaria Especial de Saúde Indígena, a qual desdobra-se em DSEl's - Distritos Sanitários Especiais, CASAl's - Casa do Índio e Departamentos para alcançar as especificidades que possui a saúde do indígena.

O que se propõe é demonstrar que a saúde indígena não é apenas política pública, mas um ato necessário em reconhecimento, e que a interferência da cultura do ser comum alcança maleficamente a saúde tradicional do índio, demonstrando que a legislação atual precisa melhorar seu arcabouço para possibilitar melhor estrutura e eficiência às atividades em desenvolvimento.

O ensaio se desenvolveu através de pesquisa mista quali-quantitativa, por procedimento bibliográfico, que a sua transcrição obedeceu ao método dedutivo, para não influenciar o leitor a um possível posicionamento político e ou partidário, mas sim, para possibilitar a compreensão do modelo de saúde aplicada aos povos indígenas.

Objetiva-se demonstrar que as Políticas Públicas, em especial a aplicada à Saúde Indígena, depende dos atos do Governo Federal, verificando sempre as modificações administrativas acerca da Secretaria Especial de Saúde Indígena 
(SESAl), bem como na continuidade dos atos em prol dos povos indígenas, não apenas saúde.

A saúde indígena é uma questão sensível, complexa, pois vincula-se à problemática da política pública indigenista, pois os povos pré-colombianos são frágeis as intempéries, enfermidades, doenças, em decorrência do não-indígena, que por razões de logística e estrutura, são vítimas de doenças com difícil combate, problemas respiratórias, doenças sexualmente transmissíveis, malária, hepatite, febre amarela, diabetes, obesidade, alcoolismo, tabagismo, entre outros.

\section{DA SAÚDE INDÍGENA}

Os povos indígenas são cidadãos e possuem todos os direitos comuns do brasileiro, bem como direitos especiais instituídos pela Constituição a exemplo da proteção especial às terras, à sua organização social (costumes, tradições e crenças - art. 231 da CF/88).

Aliás, a CF/88 também define que a saúde além de direito é dever do Estado, o que ao final completa-se com a existência do Sistema Único de Saúde (SUS - art. 196 da CF/88), que em razão dessa premissa, foi criado um subsistema aplicado à saúde do índio (Lei no 9.836/99), denominado Lei Arouca, que instituiu a necessidade da criação de Distritos Especiais Indígenas (DSEl's), que em sua configuração está a prestação de serviços relativos ao melhor atendimento dos povos, primando pela mantença da sua autodeterminação, bem como obedecendo os critérios geográficos da ocupação das etnias e os fatores culturais.

A Lei Arouca acabou surgindo para buscar dar efetividade à Política Nacional de Atenção à Saúde dos Povos Indígenas, visando:

"(...) garantir aos povos indígenas o acesso à atenção integral à saúde, de acordo com os princípios e diretrizes do Sistema Único de Saúde, contemplando a diversidade social, cultural, geográfica, histórica e política de modo a favorecer a superação dos fatores que tornam essa população mais vulnerável aos agravos à saúde de maior magnitude e transcendência entre os brasileiros, reconhecendo a eficácia de 
sua medicina e o direito desses povos à sua cultura (...)." (BRASIL, 2002, p. 8).

Para garantir a atenção à saúde acima descrita, foram instituído diretrizes que visam orientar o desenvolvimento dos trabalhos aplicáveis aos índios através de instrumentos de planejamento, implementação, avaliação e controle das ações à saúde desses povos.

- organização dos serviços de atenção à saúde dos povos indígenas na forma de Distritos Sanitários Especiais e PólosBase, no nível local, onde a atenção primária e os serviços de referência se situam;

- preparação de recursos humanos para atuação em contexto intercultural;

- monitoramento das ações de saúde dirigidas aos povos indígenas;

- articulação dos sistemas tradicionais indígenas de saúde;

- promoção do uso adequado e racional de medicamentos;

- promoção de ações específicas em situações especiais;

- promoção da ética na pesquisa e nas ações de atenção à saúde envolvendo -comunidades indígenas;

- promoção de ambientes saudáveis e proteção da saúde indígena;

- controle social. (FUNAI, 2011, p. 1)

A saúde indígena, dentro do organograma, só é possível pela sua individualização em razão das questões específicas do corpo do indígena.

\section{DO SUBSISTEMA, DA SECRETARIA E DOS ÓRGÃOS DE ATENÇÃO À SAÚDE INDÍGENA}

Neste sentido a Secretaria Especial de Saúde Indígena - SESAI (responsável por coordenar e executar a Política Nacional de Atenção à Saúde dos Povos Indígenas e todo o processo de gestão do Subsistema de Atenção à Saúde Indígena - SasiSUS no Sistema Único de Saúde - SUS), possui a missão de implementar de forma intermitente à saúde comum um modelo de gestão descentralizado, mas com autonomia administrativa, orçamentária, financeira e responsabilidade sanitária, no 
qual produz em todo o Território Nacional 34 Distritos Sanitários Especiais Indígenas - DSEl's visando estimular a atenção à saúde do indígena em seu local base, respeitando a língua mãe, sua tradição pelas práticas de saúde tradicional indígenas, bem como por fomentar o conhecimento e utilização da prática sanitária moderna pelas edificações nas aldeias de espaços de saúde, higiene, alimentação e repouso/estadia.

O que propôs foi a regulação da saúde como ato especial para fins de direcionamento pelo poder público aos povos indígenas, conforme tem-se no texto abaixo.

Conforme citado na Conferência de Proteção à Saúde do Índio, o relatório final apontou diretrizes relativas à questão da organização, pelo poder público, dos serviços de saúde para as comunidades etnicamente diferenciadas. Dentre elas destacam-se a participação de representantes indígenas na formulação de políticas e execução das ações e serviços; a criação de uma agência específica para assuntos de saúde indígena no âmbito do ministério responsável pela coordenação do SUS e o desenvolvimento de estratégias de atenção primária a nível local que respeitem as especificidades etnoculturais. Retomaremos algumas destas proposições, consideradas como ponto de partida para a discussão da integração da assistência aos indígenas às redes, de serviços do Sistema Único de Saúde. (CONFALONIERI, 1989).

Para isso é necessário entender como funciona o trabalho de assistência e atendimento à saúde indígena, fator que inicia-se pela estrutura dos Distritos Sanitários Especiais Indígenas - DSEl's, os quais conta com postos de saúde e também com Polos-base, além das Casas de Saúde Indígena (Casais), comumente conhecida como Casa do Índio, órgãos independentes entre si, mas que constituem a rede de serviços e se organizam com foco no trabalho dentro das aldeias, desenvolvido por equipe multidisciplinar e com atuação dos Agentes Indígenas de Saúde (AIS), estes vinculados a um determinado Posto de Saúde (estrutura simples, normalmente com $30 \mathrm{~m}^{2}$ ), equivalente às Unidades Básicas de Saúde - UBS atrelados a Estratégia de Saúde da Família - ESF, servidas por médicos de forma itinerante, via de regra pelo Programa Mais Médicos, e por odontólogos, enfermeiros e técnicos de enfermagem (Equipe Multidisciplinar de Saúde Indígena - EMSI), 
sendo os últimos de forma alternada entre trabalho in locus na aldeia e na unidade base em determinado município, visando o desempenho de:

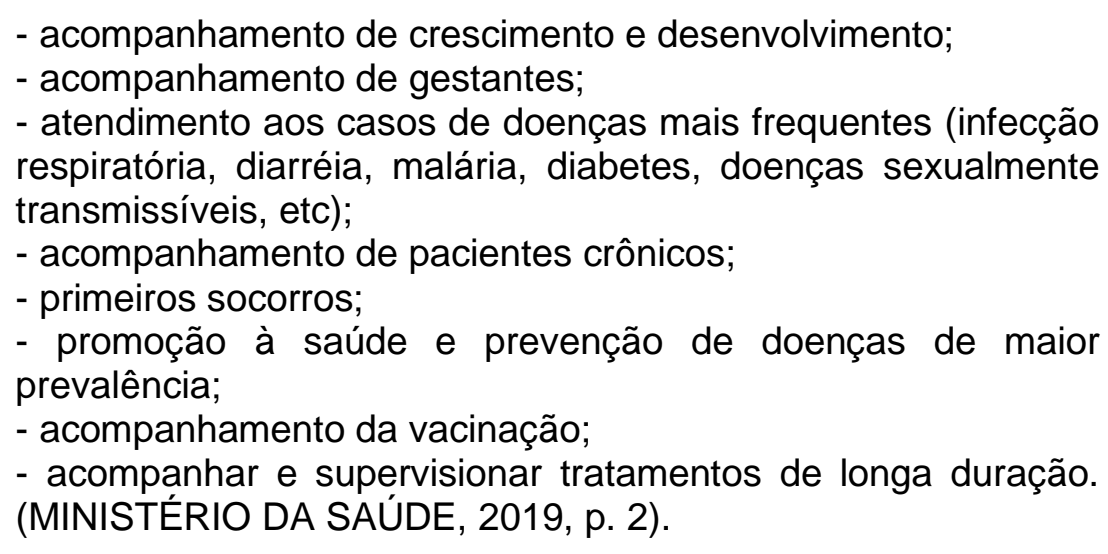

Neste sentido o Polo Base, que se divide em Polo Base I e Polo Base II, é o primeiro nível de atenção à saúde do indígena, sendo que o Polo I está localizado em Terras Indígenas (Aldeias) e o Polo II no município base de referência da zona territorial da referida Aldeia (Polo Base I).

O Polo Base I serve para prover a capacitação, reciclagem e supervisão dos AIS e demais profissionais, bem como provém pela EMSI a coleta de material para exame, além dos trabalhos de imunização, investigação epidemiológica e prevenção doenças comuns aos indígenas. Já o Polo Base II é uma estrutura física responsável pelo apoio técnico do Polo Base I, bem como para servir de aparato para com o armazenamento de medicamentos e produtos, logística, sistematização de dados, planejamento de ações, elaboração de relatório, logo a operacionalização e conclusão de toda a atividade, com campo de administração.

Observa-se que as demandas não suportadas pelas equipes multidisciplinares no campo de atuação só serão trasladadas para outro segmento, se insuficiente em sua atuação, que com atenção à regra constitucional, será o Município (OS - Pronto Socorro ou UPA - Unidade de Pronto Atendimento ou CESP Centro Especializado de Saúde Pública) quem receberá a demanda de atendimento em triagem especial, com preferência sobre os demais casos, e em sua substituição, nos locais com disponibilidade, os Hospitais Regionais, de ordem e controle da unidade da Federal (Estado ou Distrito Federal), e somente em unidades com 
estrutura federal, sob referência, haverá o atendimento, resguardado a complexidade da demanda.

Nestes casos, quando o tratamento foge à localidade da aldeia, sendo necessário o traslado do indígena para a cidade, a CASAI - Casa de Saúde do Índio (Casa do Índio) servirá de local para recepção do indígena que veio referenciado da aldeia (Polo Base I) para facilitar o acesso ao atendimento secundário e terciário (unidades comuns de saúde - PS, UPA, CESP, entre outras), possuindo as seguintes ações de apoio:

- mecanismos de referência e contra-referência com a rede do SUS;

- serviço de tradução para os que não falam português;

- realização de contra-referência com os Distritos Sanitários e articulando o retorno dos pacientes e acompanhantes aos seus domicílios, por ocasião da alta;

- recebimento de pacientes e seus acompanhantes encaminhados pelos DSEI;

- fornecimento de alojamento e alimentação dos pacientes e seus acompanhantes, durante o período de tratamento;

- prestação da assistência de enfermagem aos pacientes póshospitalização e em fase de recuperação;

- acompanhamento dos pacientes para consultas, exames subsidiários e internações hospitalares. (MINISTÉRIO DA SAÚDE, 2019, p. 2).

Dessa situação, especifica-se que as Equipes devem ter em seu currículo uma introdução base sobre a saúde indígena, fator que se faz necessário o conhecimento por estudo dirigido sobre os conceitos antropológicos, conhecimento do perfil epidemiológico da região, de atuação e também capacidade pedagógica para com as interrelações entre a Equipe, Agentes e os povos indígenas de cada etnia.

Da atenção à saúde básica, a questão antropológica aponta que quando determinada doença se dá em consequência natural da saúde do indígena, como fato comum, originário, a Equipe deve apoiar e permitir que o 'pajé' promova o trato, servindo a Equipe de base para com a melhoria da qualidade do tratamento, mas quando a doença é dita como sendo 'do branco', comum da pessoa não indígena, a Equipe deve intervir, permitindo apoio e acesso do 'pajé' em decorrência do fator cultural, mas também para a sua qualificação de fato para possíveis casos futuros, 
por isso a união da cultura indígena entre os líderes e as Equipes, formam um canal que visa aperfeiçoar ações para combater problemas de saúde, bem como conscientizar os povos indígenas para com a prevenção, melhorando a compreensão entre os atores desse processo (cultura ocidental e cultura indígena), como fonte de adesão ao invés de exclusão.

Nesta seara o que precisa ser provido para fins da saúde do indígena é a situação da capacitação para evitar a transculturação em razão do envolvimento da cultura ocidental com a cultura indígena tradicional, visando a preservação das características naturais, originárias, evitando conflitos, para compatibilizar os pilares prevenção - promoção - recuperação.

A legislação busca dinamizar a saúde de forma a integrar a relação cultural através da distritalização (criação de Distritos - DSEl's), bem como em: manter de forma complementar as práticas habituais dos indígenas e prover o treinamento especial dos Agentes e Profissionais não indígenas para com o conhecimento das etnias e suas interações, para estabelecer diferentes formas de articulação dos processos de saúde sem impactar a comunidade indígena.

Sendo assim, o que busca sustentar a legislação é a equalização em face do reconhecimento de que a cultura ocidental feriu a cultura indígena no tocante os hábito pelas interferências para buscar estabilizar a saúde indígena pelos fatores levantados em decorrência do controle social averiguado pela baixa dos níveis de desenvolvimento, isso ainda no período da constituição da Lei Arouca.

Por fim, dos parâmetros apontados, verificado a insuficiência das atividades da saúde, tem-se na legislação (art. 129, V, da CF/88) a definição de competência auxiliar do Ministério Público para defender judicialmente os direitos e interesses das populações indígenas, sem prejuízo da representatividade dos interesses políticos e sociais aplicáveis à FUNAI, pois com base no art. 200, III, da CF/88, deve o Governo o "ordenar a formação de recursos humanos na área de saúde" justamente para integrar o que propõe a Política Nacional, não apenas controle das equipes, mas da real conscientização do sistema de saúde em si.

A reportagem publicada no site EL PAIS Brasil em 31 de junho de 2019, corroborou a evolução da saúde indigenista, conforme trecho da publicação. 


\begin{abstract}
A conquista de um atendimento diferenciado.
Há 20 anos, o movimento indígena conquistou um subsistema de saúde mantido pela União que levasse em conta as particularidades étnicas, culturais e epidemiológicas de cada um dos 305 povos indígenas que vivem no país. O respeito às tradições de cura de cada povo, por exemplo, deve ser incorporado ao atendimento público. Uma secretaria especial coordena ações para os atendimentos que são realizados nos 34 distritos sanitários que funcionam nas comunidades. Essa estrutura - que já integra o SUS, mas tem especificidades próprias - trouxe avanços no acesso aos serviços de saúde, embora ainda tenha o desafio de conseguir fixar profissionais e integrá-los aos conhecimentos e crenças das etnias. No início do ano, o ministro Mandetta chegou a criticar esse subsistema ao considerá-lo "paralelo" ao SUS e disse avaliar o repasse de parte dos serviços de saúde indígena a estados e municípios. (JUCÁ, 2019).
\end{abstract}

A pesquisadora Ana Lúcia Pontes, da Escola Nacional de Saúde Pública Sergio Arouca, na mesma reportagem disponibilizada pelo site EL PAIS Brasil (2019), afirma que "o presidente Bolsonaro vem defendendo políticas integracionistas para esses povos" e menciona que "o subsistema foi criado para responder à especificidade da saúde indígena, que envolveria estratégias diferentes para planejar desde o orçamento até os vínculos dos profissionais e a atribuição da equipe de atendimento", que em resumo o Governo, através do Ministério da Saúde, tem provido pauta para melhorar 0 atendimento aos povos indígenas, para garantir os níveis de complexidade de a saúde possui, visando as complexidades, cultura, fatores epidemiológicos, distribuição territorial, sem desconsiderara a organização social, além das práticas tradicionais.

Em todo o processo, extrai-se que o Governo visa manter a base dos povos indígenas, mas como há influência do oriente no ocidente, definição jurídica para demonstrar a interferência do homem em face dos povos indígenas, também pontua a necessidade de integrar medicina alternativa. 


\section{FUNDAÇÃO NACIONAL DO ÍNDIO}

A FUNAl é uma fundação pública, possui característica de autarquia, é parte da administração pública, mas descentralizada, é o único órgão indigenista oficial com autonomia e independente funcional, mas vinculado ao Ministério da Justiça, sendo responsável pela coordenação de ações da União Federal em face das garantias constitucionais aplicáveis aos povos indígenas, a exemplo do território através de política de demarcação e fiscalização, além dos projetos sustentáveis e de manejo aos povos indígenas, bem como àqueles aplicados à saúde.

Desde o ano de 1967, várias instituições se responsabilizam pelo atendimento à saúde indígena e também ao próprio indígena. A FUNAI não se vincula apenas à saúde, mas todas as relações de direitos e obrigações atinentes aos povos indígenas, com caráter difuso, a exemplo da Secretaria Especial de Saúde Indígena, terceirizados e até o Ministério Público Federal (MPF), entre outros.

No que concerne a atenção da saúde, deve-se valorar o que define o Decreto no 7.778 , de 27 de julho de 2012, pois exige que a FUNAI monitore as ações de serviços aplicados à saúde como forma de ferramenta de defesa, pois a fiscalização visa garantir, efetivar, as ações de saúde em desenvolvimento, reduzindo os prejuízos e perdas na saúde.

Mas o papel da Fundação, vem sendo desenvolvido? Para isso, como forma de controle, pela teoria constitucionalista, tem-se o papel do Ministério Público Federal como contra peso ao sistema, servido de apoio aos atos institucionais e de competência da FUNAI, mas também como parte, custus legis, para tratamento de representação e denúncias. 


\section{DA ATUALIDADE E DO FATOR POLÍTICO}

Da demonstração do funcionamento da saúde pública no país, tem-se que é imprescindível valorar também as mudanças políticas no campo da saúde, em especial a saúde indígena.

Recentemente, o atual Presidente da República, em resposta ao movimento indigenista internacional, disse que as formatações administrativas que ocorreram buscam dar eficiência às políticas para que haja o continuísmo das ações aplicadas à saúde dos povos indígenas, como ato de desenvolvimento nacional em respeito às relações internacionais e compromissos assumidos pelo Brasil em gestões anteriores.

O Governo atual, além de manter a SESAI, sinalizou que alterações de caráter administrativo foram e são necessário para poder dar eficiência às ações, como ocorreu pelo Decreto o 9.795, de 17 de maio de 2019 (Aprova a Estrutura Regimental e o Quadro Demonstrativo dos Cargos em Comissão e das Funções de Confiança do Ministério da Saúde, remaneja cargos em comissão e funções de confiança, transforma funções de confiança e substitui cargos em comissão do Grupo-Direção e Assessoramento Superiores - DAS por Funções Comissionadas do Poder Executivo - FCPE), o qual alterou a sistemática funcional da Secretaria Especial de Saúde Indígena, no qual manteve-se na expectativa da manutenção da atividade, em razão da ideia de integrar a saúde indígena ao sistema único de saúde, em razão da atuação dos municípios, visando dar autonomia gradual e liberdade econômica futura aos gestores dos municípios nos atendimentos aos povos indígenas, enquanto saúde.

Do apontamento, a única controvérsia que surgiu, foi a extinção do Departamento de Gestão da Saúde Indígena (órgão responsável pela garantia das condições necessárias à gestão do subsistema, através da programação da aquisição de insumos para as unidades de atendimento), no qual agregou à Secretaria a função dentro de sua estrutura, fato que visou a economicidade, mas não valorou a eficácia da alteração, até a presente data. 


\subsection{PROBLEMAS X EXPECTATIVA}

A base histórica indígena deve ser analisa a partir da atuação da FUNASA (Fundação Nacional de Saúde) que estava a frente em 1999 da Descentralização Sanitária, para buscar combater a desnutrição e problemas de saúde vinculados à ausência de controle sanitário, esgotos e água tratada, que surgiu como fator positivo, dentro da expectativa em tutela à saúde dos povos indígenas.

Mas em meados de 2005, conforme reportagem extraída do portal do Instituto Socioambiental (2018) "houve uma explosão nos protestos de diferentes etnias em todo o Brasil, revelando situações de abandono e descaso no atendimento das populações indígenas", surgindo uma investigação com resultado em que demonstrou que a legislação não era eficaz e, que, os óbitos decorriam da ausência de alimento e água potável, em sua maioria, sendo que em 2006, conforme relatado, o Ministério Público Federal "criou um grupo de trabalho para investigar problemas com os convênios firmados com as organizações que realizam o atendimento local", surgindo o conhecimento da burocratização construída de forma diversa à da Política Pública, para modelar a forma de contratação, aquisição e tratamento, em detrimento ao bem jurídico tutelado, saúde indígena.

A proposta do ensaio é apresenta que existe legislação, um marco regulatório, que possibilita o avanço das questões aplicadas à saúde, razões que nos dá base da evolução, lenta, mas necessária, das ações governamentais aos povos indígenas, sendo o censo e contra censo a questão da problemática e expectativa apontada, apenas em um dos pontos, para basilar e quantificar que 0 formato da legislação é intangível, dependendo da modulação do ser para a produção do resultado a que se propõe a saúde indígena, logo, deve-se haver posição crítica reflexiva para que todos possam ser atores dessa atividade, propositores, fiscais e parceiros, todos nesse novo modelo de gestão que se apresenta com base na legislação. 
Sobre o apontamento em que existe dúvidas e certezas, o que absorve enquanto resultado deste, é a reorganização do sistema que visa integração e distribuição de serviços e, que se apresenta como fator construtivo da nova estrutura da saúde aos povos indígenas.

Essa distinção pode ser auferida pela manifestação do escritor RIBEIRO em sua obra "Os Índios e a Civilização" no ano de 1982, antecedente à modernização administrativa, conforme segue:

Da mesma forma, na necessária reestruturação administrativa ministerial para a adequada gestão do Sistema a nível central, surge a oportunidade para a criação de um organismo específico para a coordenação executiva das ações de saúde e responsável pela elaboração e implementação de políticas de saúde para as populações indígenas. Estas embora se constituam em apenas $0,2 \%$ da população do país, apresentam, como um todo, um crescimento demográfico acima daquele observado na população não-índia, podendo-se prever um aumento rápido na demanda de atenção médicosanitária. Nos períodos de estabilidade demográfica que se seguiram às de populações pós-epidêmicas iniciais, alguns grupos exibiram crescimento numérico acelerado, da ordem de $500 \%$ em duas décadas (RIBEIRO, 1982).

Extrai-se do texto uma visão da década de 80 (oitenta) em que o controle populacional era fragilizado e os problemas decorrentes da saúde não se tinha resultados hábeis, que dessa evolução, 20 (vinte) anos de saúde indígena, com subsistema, ainda se tem um enorme desafio para o desenvolvimento efetivo das ações em prol da saúde indígena.

André Antunes (2019) em publicação de sua pesquisa - 20 anos de saúde indígena no SUS - expôs, sobre essa perspectiva, apresentou com ótica isolada um ponto essencial, que é base de ser replicado em outras unidades em razão das circunstâncias da situação da saúde indígena.

\footnotetext{
Conjuntura adversa

Vinte anos após a aprovação da lei que criou o Subsistema, sua consolidação permanece um desafio para os povos indígenas e o SUS, especialmente em uma conjuntura em que vários direitos desses povos vêm sendo questionados. No final de setembro, em seu discurso de abertura na Assembleia Geral das Nações Unidas, o presidente Jair Bolsonaro criticou a extensão das terras indígenas demarcadas no país e garantiu
} 
que não serão demarcadas novas terras. A delegação brasileira fora à Nova York acompanhada de Ysani Kalapalo, indígena apoiadora do governo apontada como representante dos povos do Xingu, mas cuja representatividade foi questionada em uma carta de repúdio assinada por lideranças da região. (ANTUNES, 2019).

O autor afirmar que não existe solução sem problema, havendo um princípio formador na origem da condição política, pois sem o reconhecimento antrópico, não haverá reconhecimento para a aplicação e desenvolvimento da saúde, logo, meio ambiente.

\section{CONSIDERAÇÕES FINAIS}

O texto confirma o que se propõe, qual seja, demonstrar a ferramenta legal conforme sua real constituição enquanto saúde indígena sob a referência da legislação de forma prática para sustentar a demonstração do programa jurídico brasileiro aplicado enquanto teoria sob o princípio da eficácia à saúde dos índios, visto que atualmente $\mathrm{o}$ atendimento se faz pelo sistema público através de intermediários.

Busca-se promover a discussão da relação saúde e etnias enquanto fator cultural juspositivado, fato desenvolvido por uma cadeia de profissionais de interdisciplinas e, que, se dá através de comissões técnicas e de órgãos governamentais.

Conclui-se que o Governo, como gestor da coisa pública, precisa efetivar as políticas públicas formuladas em razão dos indígenas, bem como deve incorporar gradativamente as discussões em todos os setores, educação, ensino e prática prevenção, promoção e recuperação, visando o fortalecimento com base no conteúdo cultural original dos povos indígenas, pois além de fato fundamental, é prioridade em face de suas peculiaridades, para integrar a saúde indígena como um ato social como um todo e não como exclusivo e restrito. 


\section{REFERÊNCIAS BIBLIOGRÁFICAS}

ANTUNES, André. 20 anos de saúde indígena no SUS. Publicado em: 21/11/2019. Disponível em: $\quad$ http://www.epsjv.fiocruz.br/noticias/reportagem/20-anos-de-saude-indigena-no-sus>. Acesso em: 30/03/2020.

BRASIL. Constituição da República Federativa do Brasil de 1988. Disponível em: <http://www.planalto.gov.br/ccivil_03/constituicao/constituicao.htm>. Acesso em: 20/01/2020.

Política Nacional de Atenção à Saúde dos Povos Indígenas. Elaborado em Brasília/DF, março de 2002. Disponível em: < http://bvsms.saude.gov.br/bvs/publicacoes/politica_saude_indigena.pdf >. Acesso em: 20/01/2020.

Saúde Indígena. Disponível em: <https://pib.socioambiental.org/pt/Sa\%C3\%BAde_Ind\%C3\%ADgena>. Acesso em: 05/02/2020.

CONFALONIERI, Ulisses E. C. O Sistema Único de Saúde e as populações indígenas: por uma integração diferenciada. Cadernos de Saúde Pública out/dez 1989. vol.5 ํㅜ․ ISSN 0102-311X. On-line version ISSN 1678-4464. Disponível em: <https://doi.org/10.1590/S0102311X1989000400008>. Acesso em: 01/02/2020.

DESCRITORES EM CIÊNCIAS DA SAÚDE. DeCS. ed. rev. e ampl. São Paulo: BIREME / OPAS / OMS, 2017. Disponível em: < http://decs.bvsalud.org >. Acesso em: 30/03/2020.

FUNAI. Saúde. Elaborado em 2011. Disponível em: <http://www.funai.gov.br/index.php/saude>. Acesso em: 20/02/2020.

INSTITUTO SÓCIO AMBIENTAL. Saúde Indígena. Publicado em 27/08/2018. Disponível em: < https://pib.socioambiental.org/pt/Sa\%C3\%BAde_Ind\%C3\%ADgena>. Acesso em: 30/03/2020.

JUCÁ, Beatriz. Decreto de Bolsonaro com mudanças na saúde indígena dispara alerta no movimento indigenista. Publicado em 31/06/2019. Disponível em: $<$ https://brasil.elpais.com/brasil/2019/05/30/politica/1559238132_162541.html >. Acesso em: 30/03/2020.

MINISTÉRIO DA SAÚDE. Saúde Indígena. Publicado em 2019. Disponível em: $<$ https://www.saude.gov.br/saude-indigena>. Acesso em: 22/02/2020.

NEVES, Úrsula. Como é o trabalho de assistência na saúde indígena. Disponível em: $<$ https://pebmed.com.br/como-e-o-trabalho-de-assistencia-na-saude-indigena/>.

RIBEIRO, D. Os Índios e a Civilização. Petrópolis: Vozes, 1982. 